\title{
Thermal-Electrochemical Lithium-Ion Battery Simulations on Microstructure and Porous Electrode Scale
}

\author{
A. Latz ${ }^{\text {abc }}$ and J. Zausch ${ }^{\mathrm{d}}$ \\ ${ }^{\text {a }}$ Institute of Engineering Thermodynamics, German Aerospace Centre (DLR), 70569 \\ Stuttgart, Germany \\ ${ }^{\mathrm{b}}$ Helmholtz Institute Ulm for Electrochemical Energy Storage, 89081 Ulm, Germany \\ ${ }^{\mathrm{c}}$ Institute of Electrochemistry, University Ulm, 89069 Ulm, Germany \\ ${ }^{\mathrm{d}}$ Fraunhofer Institute for Industrial Mathematics, 67663 Kaiserslautern, Germany
}

\begin{abstract}
Heat production within a battery cell is governed by local fluxes of ions and electrical currents in the microstructure of the battery electrodes. These fluxes are strongly coupled with thermal transport, leading to a fully coupled reaction transport model for batteries [1-3]. By applying the volume-averaging technique to our thermal-electrochemical microscopic theory the corresponding thermal-electrochemical porous electrode theory can be derived [3]. Implementation of both models in our simulation software BEST [4] allows to compare the results of the homogenized theory with numerically averaged simulations on micro scale. Whereas the numerically averaged results agree very well with the results of porous electrode theory, strong fluctuations around this average (e.g. of the overpotential) are observed in microstructures. Our results raise the question to which extent porous electrode theory can be used to predict degradation phenomena in batteries.
\end{abstract}

\section{Introduction}

In the pioneering work of Newman and coworker [5-8] a porous electrode theory for Li ion batteries was established, which allows the simulation of $\mathrm{Li}$ ion distribution and potentials with the corresponding ion fluxes and electrical currents in a battery cells. The simulations are mostly in a 1D+1D setting i.e. the phenomena in a $1 \mathrm{D}$ cell model in which electrodes are treated as effective porous media are coupled to diffusion in a representative particle at each point of the electrodes. The models can be justified and derived with the help of homogenization techniques as e.g. volume averaging [9-10] or formal mathematical homogenization theory [11]. The original models of Newman and coworkers have been complemented by thermal transport [12-14] and are also used in the analysis of degradation phenomena as e.g. plating [15]. In recent years models and simulations are going to be extended to the microstructure scale [3, 16-19]. On this scale transport has to be simulated for each active particle coupled to the transport in the electrolyte by interface reactions at each spatial point of each particle. In order to have confidence that the simulations can reproduce realistically phenomena on the microstructural scale and to ensure thermodynamic consistency they have to be based on a rigorously derived theoretical structure. This structure was developed in $[1,3]$. There an 
extensive discussion of the assumptions of the theory and the relation to other microstructural simulations can be found. In order to study the similarities and differences of microstructure based theories and porous electrode theory, in [3] the fully coupled electrochemical-thermal porous electrode theory was derived by applying volume averaging techniques to the theory in [1]. In the following we describe the results of the simulations presented in [3] for the limits of porous electrode theory to predict local phenomena relevant for the accurate description of degradation phenomena in Lithium ion batteries.

\section{Simulation details}

In order to demonstrate principal differences between microscopic and homogenized meso-models, mostly based on porous electrode theory with respect to thermal aspects, we performed numerical computer simulations on a generic model system with two different micro structures and compared the results to meso-scale simulations of two corresponding porous electrode models.

To demonstrate the qualitative features of the microscopic electrochemical model no measured electrode structure was used but rather a computer-generated random geometry with typical properties. To simplify the geometry further both electrodes have an identical structure. Two cases with different base particles were considered: one with spherical active particles of radius $5 \mu \mathrm{m}$ and one with prolate spheroids of random orientation with half-axes of $5 \mu \mathrm{m}$ and $16.8 \mu \mathrm{m}$. In both cases the porosity $\varepsilon$ was set to 0.5 such that the capacity of each electrode is equal. The geometries are shown in Fig1. Left and right electrodes are anode and cathode, respectively. They are connected to current collectors through which electrons enter. Note that although electrodes are equal, their interface area with electrolyte differs slightly since they are attached to the collector plates on opposite sides. The simulation was set up such that the virtual cell is almost empty and a constant current was applied to charge the cell. All simulation were performed with our simulation software BEST [2]. Details on the parametrization and on the numerical algorithms can be found in [3].

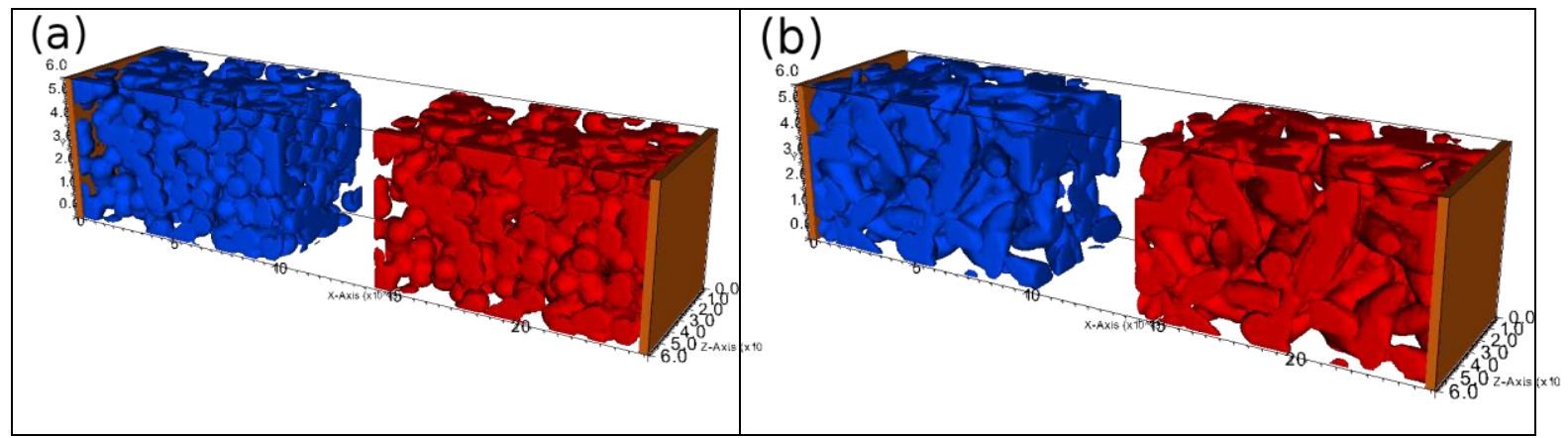

Figure 1: Geometry used for the microscopic simulation. It consists of anode (blue), cathode (red) and current collectors (brown) and electrolyte (space between particles and electrodes. Geometry (a) consists of spherical particles of radius $5 \mu \mathrm{m}$, geometry (b) of a prolate spheroids of half-axes with $5 \mu \mathrm{m}$ and $16.8 \mu \mathrm{m}$. The thickness of each electrode is $100 \mu \mathrm{m}$, the separator region $40 \mu \mathrm{m}$ and the cross section area $60 \times 60 \mu \mathrm{m}^{2}$

The geometry used for the mesoscopic simulations is shown in Fig.2. Except for the current collectors that have now a thickness of $40 \mu \mathrm{m}$, the thicknesses of electrodes and separator are the same as in the micro case. The lateral dimensions were increased to 260 $\mu \mathrm{m}$ but the applied current was scaled proportionally. The parametrization was chosen 
such that meso- and micro-simulations can be compared. To this end the effective transport properties required in the meso-case are computed from the micro-structure using the software GeoDict [4] instead of using phenomenological expressions as e.g. the Bruggeman relations.

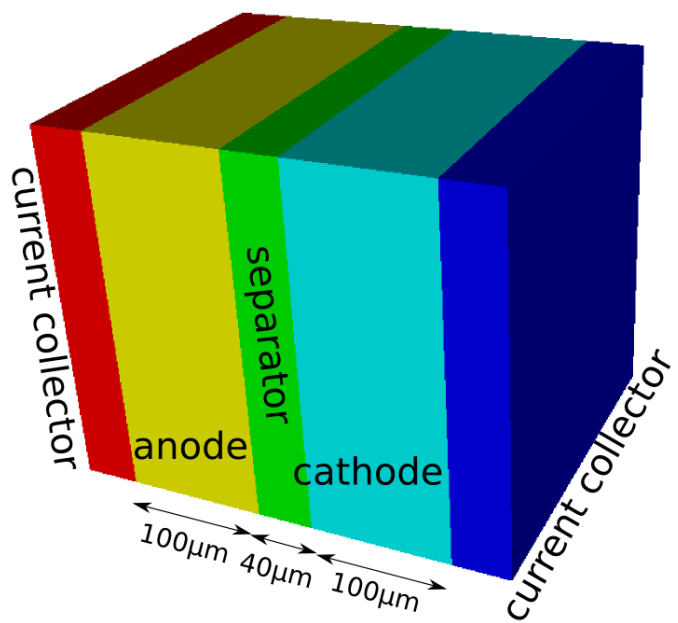

Figure 2: Geometry for the mesoscale simulations

The meso-model used here is a 3D+1D model - three spatial dimensions for the unknowns electrolyte concentration, electrolyte potential, solid potential and temperature and in each electrode CV another virtual dimension for the concentration within a representative spherical particle (solid concentration) to mimic the diffusion into the active material. As in the micro-model a finite volume discretization is used for both the $3 \mathrm{D}$ cell geometry and the $1 \mathrm{D}$ domain representing a microscopic particle. Here, each $3 \mathrm{D}$ electrode voxel contains its extra dimension for the representative particle which is spatially discretized into 10 control volumes. More details on the numerical procedure can be found in [3].

Results and discussions.

The simulation yields a three-dimensional field of lithium ion concentrations. Of interest is, for instance, the ion concentration in through-plane direction. A projection of the Li-concentration of the electrolyte phase onto this axis is shown in Fig. 3. The data from the microscopic simulations exhibit considerable fluctuations reflecting the inhomogeneous, random structure. There are even CVs that remain at their initial concentration of $1.2 \mathrm{~mol} / \mathrm{l}$ since they are surrounded completely by active particles. Due to the electroneutrality condition they have to stay at their initial concentration. The porous electrode approach treats the complete electrode region as homogeneous effective medium. For this reason (and of course due to the application of symmetric boundary conditions) the concentration profile does not show any scatter and agrees reasonably well with the microscopic average. However, a quantitative agreement is only obtained in the separator region for the sphere-based micro-geometry. Especially in the electrodes far away from the separator there is a deviation of about $5 \%$. These deviations might be related to the fact, that homogenization only yields satisfying results if the underlying 
assumption of scale separation is fulfilled. Very often electrodes have a thickness in the order of only ten particle diameters, which is not sufficient to justify the assumption of scale separation. Therefore it seems not unreasonable that for the ellipsoidal base particles the concentration in the separator region does not match well because in this case also the representative sphere of the porous electrode model is different from the actual micro-particle (ellipsoid). Thus it is important to determine the effective properties as well as the representative particle size with great care.

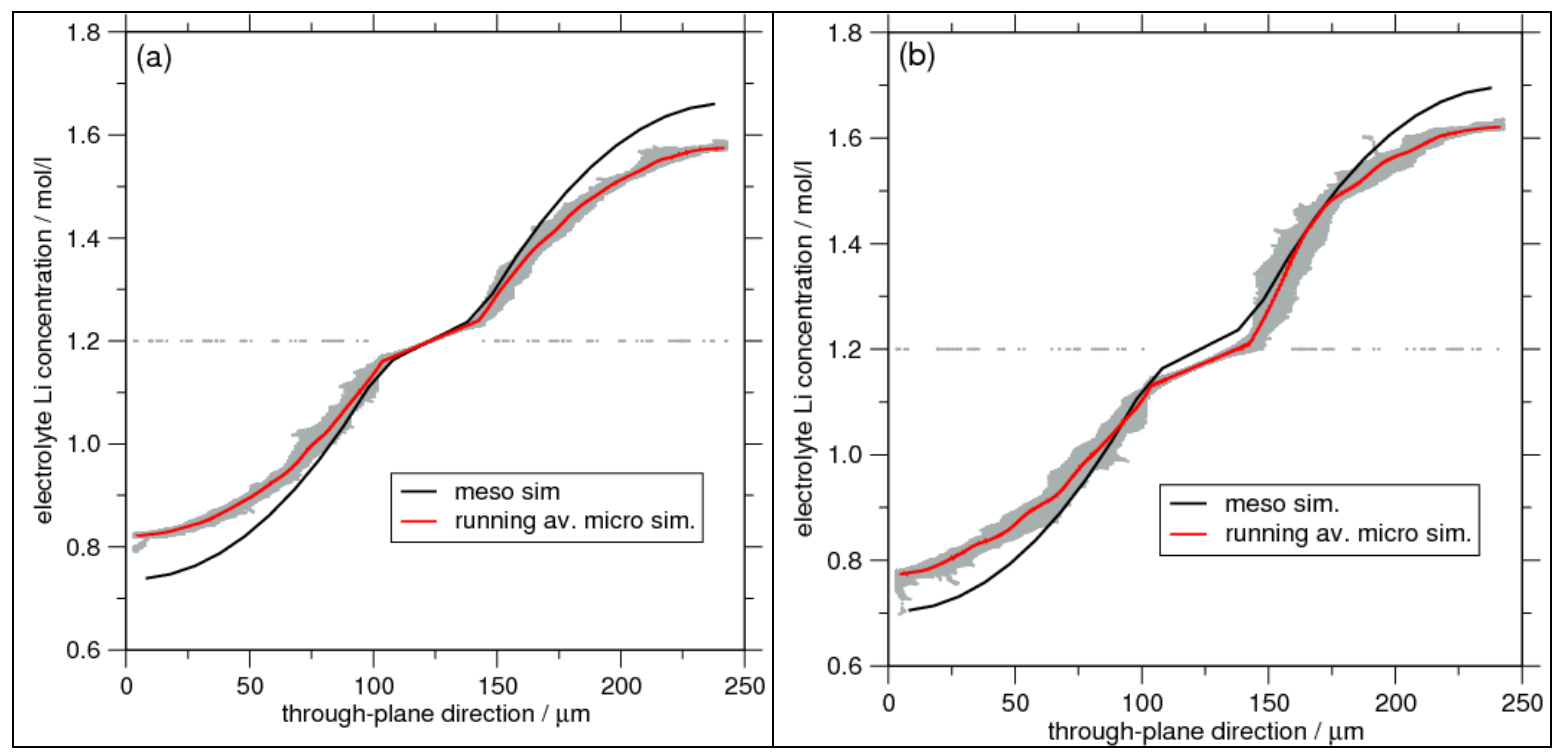

Figure 3. Distribution of electrolyte concentration as function of position in throughplane direction at capacity ratio 0.42 for (a) spherical particles and (b) prolate spheroids. Each panel compares results of the micro-simulation with a corresponding meso-scale simulation.

This is very relevant, for instance, for a prediction of the limiting current, where the electrolyte is locally depleted of ions. The concentration distribution within the active material is indirectly expressed through the cell potential (differences between current collector potential at cathode and anode). For the four simulation cases the cell-potential is shown in Fig. 4. Comparing the microscopic cell potentials it is interesting to note the higher voltage for the ellipsoidal case than for the spherical case. This is explained by the different interface areas: While the ellipsoidal micro-geometry has an interface area of $0.18 \mathrm{~mm}^{2}$, it is $0.22 \mathrm{~mm}^{2}$ for the one based on spheres. Since in both cases the same current is applied a higher overpotential (and therefore cell potential) is required for smaller interface areas. Although the meso-simulation shows the same behavior the corresponding micro- and meso-simulations differ slightly. Since the lithium diffusion within active particles is modeled in the meso-case only by single, representative particle, the exact influence of the actual interface shape and connectivity between particles is neglected. Thus it cannot be expected that both methods show a better agreement without more careful adjustment of parameters, in particular of the radius $r$ in the meso-model. However, even then it is not clear that this will lead to the same results also in other application scenarios e.g. with a different applied current. 


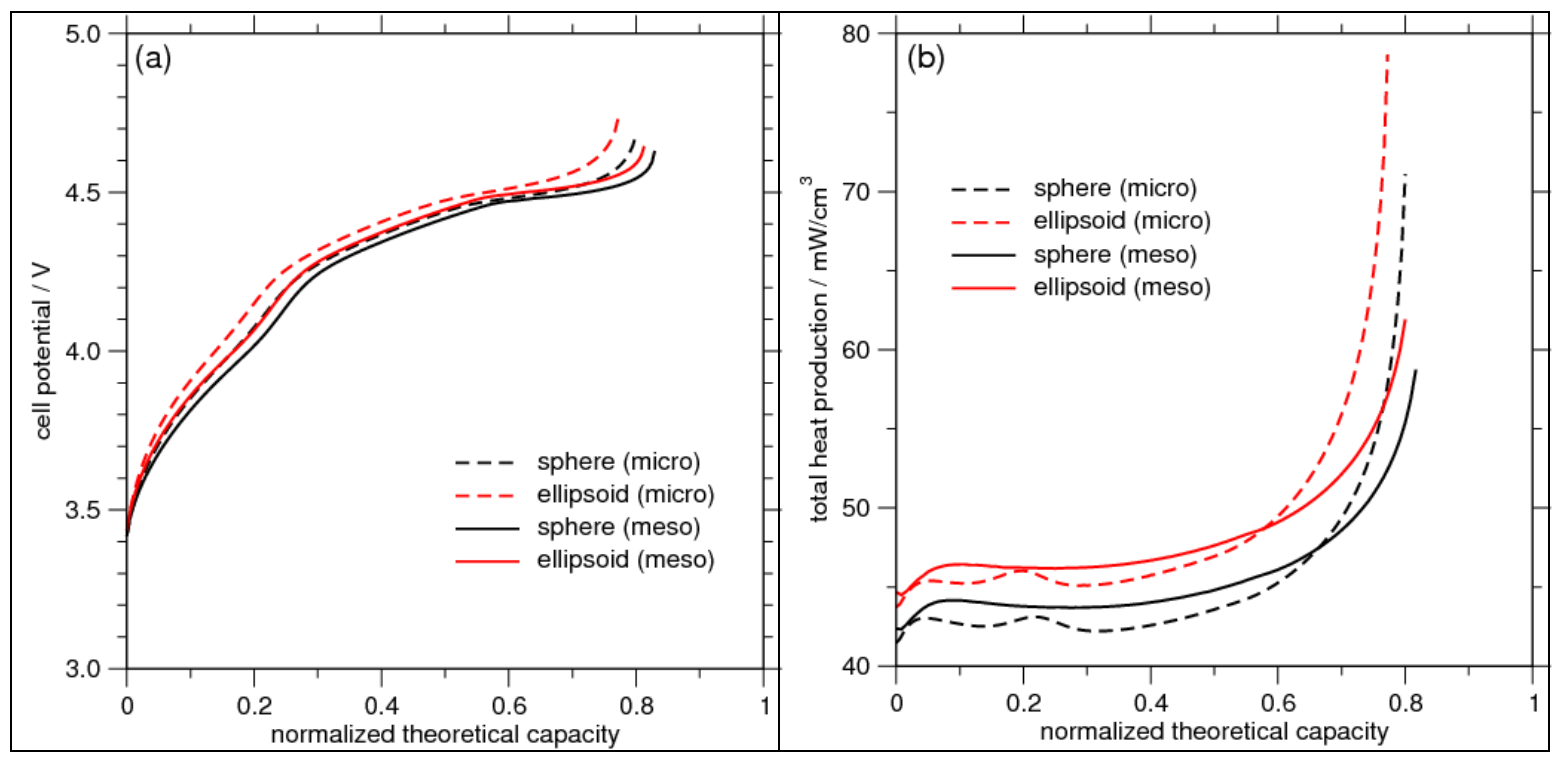

Figure 4: (a) Comparison of cell potential during charging simulations on micro- and meso-scale for two considered base-particles sphere and ellipsoid. (b) Comparison of heat production shown in a similar way as in (a).

Of particular interest in this model comparison are thermal effects. Because of the mesomodel's neglect of the structural details the current and ion flow is much different from the microscopic case. There the current can be very localized due to the morphological properties of the electrode structure. Thus it is very surprising that a comparison of the total heat power per volume is very similar for both simulation approaches (cf Fig. 4(b)). Additionally, as before, we recognize a difference of heat production between spherical and ellipsoidal base particles. As discussed in [3], it is the localized nature of the heat production, which is responsible for the differences in the heat production. In our simulation, the by far biggest contribution to the heat production is the Joule heating at the interface i.e. the product of overpotential $\eta$ with the exchange current $i_{\text {se }}$ at each interfacial point of a particle integrated over all particle surfaces. In the porous electrode model this heat enters the heat production of the representative particle as integral over all surface sources per volume. It turns out, that this average interface Joule heat can be captured in the meso-model quite well, although there is a deviation at the end of the charging process. Since the interfacial Joule heating is the biggest contribution to the heat production, similar to the cell potential, also the total heat (Fig. 4(b)) crucially depends on the overpotential. Therefore the same reason as discussed for the cell potential (Fig. 4(a)) explains the difference.

From the discussion above one would expect that the overpotentials of micro- and meso-model are the same. In Fig. 5(a) we study the overpotential at a fixed time as function of position in through-plane direction. Comparing the overpotentials for each $\mathrm{CV}$ in the microscopic case (grey dots) large fluctuations around the average (red curve) are observed. These fluctuations are due to the complex microstructure. They cannot be captured by the homogenized mesoscopic porous electrode theory (black curve), which agrees remarkably well with the numerical average of the microscopic simulation. Due to the strong correlation between interfacial Joule heating and overpotential the same holds for this heat source shown in Fig. 5(b). Based on our numerical results we can draw two conclusios. First, in the case considered in this paper, the porous electrode approach is 
capable of reproducing the average or global heat power. Second, the local deviations from the averaged theory due to the complex microstructures can be very large and are in general not negligible.

It is important to mention that the even first conclusion cannot be expected to hold universally. One should note that the good agreement is observed, because there is just one source, the interfacial Joule heat, that plays the dominant role. As long as the geometry is such that overpotential and exchange current density can be reproduced on the mesoscopic scale, porous electrode theory can compute the correct amount of heat. If other sources of heat that rely on the actual distribution of current or ion fluxes become more important either due to different material parameter combinations or geometric properties it is unlikely that a porous electrode simulation can reproduce the results of the full microscopic approach.

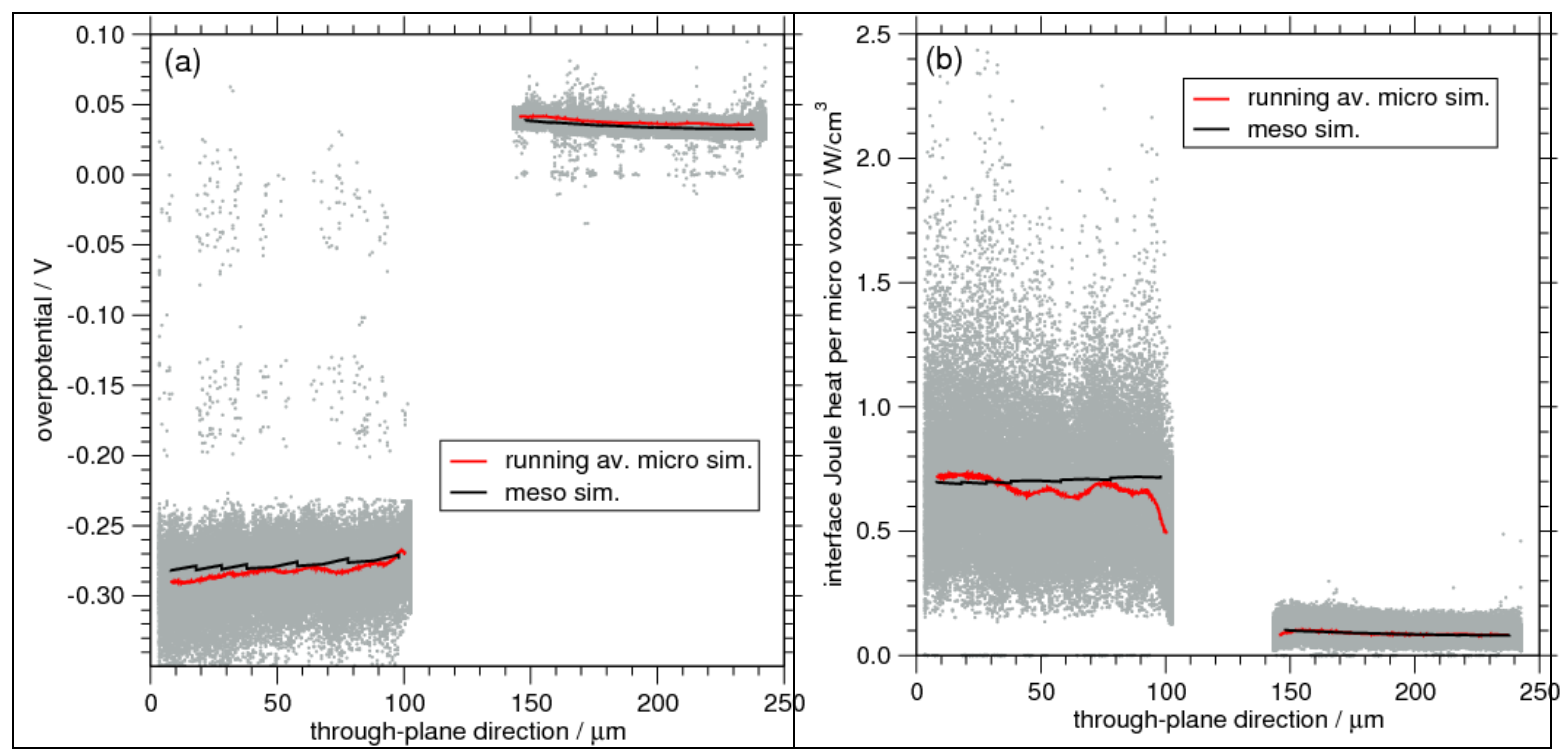

Figure 5: (a) Spatial distribution of overpotential in through-plane direction for ellipsoidbase microstructure at capacity ratio 0.42 . Due to the strong variation of the data in the microscopic simulations (grey), a running average (red) is compared to the overpotentials of the mesoscopic simulations (black). (b) Similar as in (a) we compare the Joule heat at the interface between active particles and electrolyte.

Even in cases where the porous electrode theory describes the average phenomena reasonably well, the local fluctuations are not captured. But exactly these fluctuations are potentially important for prediction of the onset of degradation phenomena. For example Lithium metal plating can potentially occur at any point of the particle surface at which the actual potential (open circuit potential + overpotential) drops below 0V against the redox potential $\mathrm{Li} / \mathrm{Li}^{+}$. The observed fluctuations of the overpotential (Fig. 5(a)) can become as large as $50 \mathrm{mV}$, which might be just enough to produce a local plating event, which in turn can be the seed of a thermal running way.

\section{Summary.}

We compared microstructural resolved simulations of coupled electrochemicalthermal phenomena in Lithium ion batteries with a porous electrode theory, which was derived from the microscopic theory with the help of volume averaging. The porous 
electrode simulation approach reproduced quantitatively the average amount of energy predicted by the microscopic approach for charging with a constant current. The good agreement could be explained by the fact that in the case considered here, there is only one dominant source of heat, namely Joule heating at particle-electrolyte interfaces. This heat term does only depend on overpotentials and intercalation currents which are reproduced in this approach reasonably well. Our simulation also show that local fluctuations of overpotentials and heat production terms due to the complicated microstructure can be quite large, in fact too large to be neglected for the prediction of degradation phenomena. It seems therefore necessary to include those fluctuations in improved porous electrode theories, to be developed.

\section{Acknowledgments}

Parts of the paper are reproduced from our article in the Beilstein Journal of Nanotechnology [3], which was published under Creative Commons Attribution License (http://creativecommons.org/licenses/by/2.0). We thank German Federal Ministry of Education and Research (BMBF) for partial financial support through the projects "TopBat", grant 16N12529 and LiEcoSafe, grant 03X4636a.

\section{References}

1. A. Latz, J. Zausch, J. Power Sources. 196, 3296-3302 (2011).

2. A. Latz, J. Zausch, Electrochim. Acta. 110, 358-362 (2013).

3. A. Latz, J. Zausch , Beilstein J. Nanotechnol. 6, 987-1007 (2015).

4. www.itwm.fraunhofer.de/best

5. J. Newman, W. Tiedemann, AIChE J. 21, 25-41 (1975).

6. M. Doyle, T.F. Fuller, J. Newman, J. Electrochem. Soc. 140, 1526-1533 (1993).

7. T.F. Fuller, M. Doyle, J. Newman, J. Electrochem. Soc. 141, 1-10 (1994).

8. J. Newman, K.E. Thomas-Alyea, Electrochemical Systems, Wiley, (2004).

9. P. De Vidtst, R.E. White, J. Electrochem. Soc. 144, 1343-1353 (1997).

10. M. Landstorfer, T. Jacob, Chem. Soc. Rev. 42, 3234-52 (2013).

11. F. Ciucci, W. Lai, Transp. Porous Media. 88, 249-270 (2011).

12. D. Bernardi, E. Pawlikowski, J. Newman, J. Electrochem. Soc. 132, 5-12 (1985).

13. C.R. Pals, J. Newman, J. Electrochem. Soc. 142, 3274-3281 (1995).

14. K.E. Thomas-Alyea, J. Newman, J. Electrochem. Soc. 150, A176-A192 (2003).

15. P. Arora, M. Doyle, R.E. White, J. Electrochem. Soc. 146, 3543-3553 (1999).

16. C.W. Wang, A.M. Sastry, J. Electrochem. Soc. 154, A1035-A1047 (2007).

17. G.B. Less, J.H. Seo, S. Han, a. M. Sastry, J. Zausch, A. Latz, et al., J. Electrochem. Soc. 159, A697-A704 (2012).

18. B. Yan, C. Lim, L. Yin, L. Zhu, J. Electrochem. Soc. 159, A1604-A1614 (2012).

19. B. Yan, C. Lim, L. Yin, L. Zhu, Electrochim. Acta. 100, 171-179 (2013). 